\title{
Research on Negotiation based Bargaining Strategies in e-Commerce
}

\author{
Jiang Jianhua ${ }^{1, a}$, Zhang Guangyun ${ }^{1, b}$, Hong Niansong ${ }^{2, c}$ \\ ${ }^{1}$ College of Computer Engineering, Guangdong Institute of Science and Technology, Zhuhai, China \\ ${ }^{2}$ College of Information and Communications, Zhejiang Industry \&Trade Polytechnic, Wenzhou, \\ China
}

a710281792@qq.com, b149546961@qq.com, '12962272@qq.com

Keywords: Negotiation, Bargaining Strategy, Quoted Price, Electronic Commerce

\begin{abstract}
As to price negotiation in e-commerce, a formal model of price bargaining negotiation was constructed. The bargaining strategies of single-issue and multi-issue were researched. In single-issue negotiation, the assessment method of quoted price's utility value was given out, and the bargaining strategy based on bargaining reincarnation number and rivals' bargaining behavior were designed respectively. In multi-issue negotiation, the bargaining strategy based on equivalent utility value was researched and the algorithm of generating quoted price with equivalent utility value was designed, the bargaining strategies in this paper can be used in transaction process of e-commerce effectively.
\end{abstract}

\section{Introduction}

Conflict of interest is common in price negotiation in e-commerce. One of the best ways is by bargaining negotiation. Ya'akov Gal thinks that negotiation is a process of compromise for participants expressing their conflicting demands firstly and then to seek solutions by mutual concessions ${ }^{[1]}$. As to theory of bargaining, Ma Benjiang defines the buyer's strategy "Be insatiable" for the problem of argy_bargy ${ }^{[2]}$. Su Qing discussed the supply chain multilateral negotiation about profit distribution between one buyer and many suppliers ${ }^{[3]}$. Wang Kanliang takes tri-stages bargaining as example, researches the problem of dynamic game of asymmetry information bargaining ${ }^{[4]}$. Zhan Wenjie researches the evolution of strategies in a many-to-many bargaining based on assumption that traders are boundedly rational ${ }^{[5]}$. In the application of bargaining, Li Yuyu proposed a bargaining cooperative game model for assemble-to-order(ATO) supply chain composed of a final product manufacturer and two suppliers ${ }^{[6]}$. Wang Yi researched the Medium and long-term power purchase plan based on bargaining game ${ }^{[7]}$. Liu Yun proposed a resource allocation algorithm based on the cooperative game theory of generalized Nash bargaining solution $^{[8]}$. The essence of bargaining negotiation is on behalf the interest of participants to seek an agreed goal or negotiation fails. So, a bargaining negotiation model was established firstly in this paper, and then the bargaining strategies in the model were researched from two aspects of single-issue and multi-issue negotiation.

\section{Formal Model of Negotiation}

According to bargaining negotiation process in e-commerce, the formal model of negotiation is defined as follow Eq. 1. $N m=<N, T, V^{T}, P, S, U, t>$

Where $N$ is participants and expressed as $N=\left\{a, b_{1}, b_{2}, \ldots, b_{r}\right\}, a$ is seller and $b$ is buyer. If $r=1$, it is one-on-one negotiation and it is one-to-many negotiation while $r \geq 2$.

$T=<T_{1}, T_{2}, \ldots, T_{n}>$ is issues of negotiation. It can be used to indicate such as cost, time, quality and etc. If $n=1$, it is a single-issue negotiation, it is multi-issue negotiation where $n \geq 2$.

$V^{T}=<V^{T_{1}}, V^{T_{2}}, \ldots, V^{T_{n}}>$ is the value of issues $T$.

$P$ is protocol of negotiation, it is used to control and manage the interaction rules of 
negotiation , such as the protocol of stipulating participants must bid at the same time or bid in turn.

$S$ is strategy refers to the actions taken by participants to achieve their goals in the process of negotiation , such as concession strategies or win-win strategy.

$U=\left\{U_{a}, U_{b_{1}}, U_{b_{1}}, \ldots, U_{b_{r}}\right\}$ is the utility value of participants.

$t$ is reincarnation number of negotiation and $0 \leq t \leq t_{\max }, t_{\max }$ is the deadline or maximum reincarnation number.

\section{Bargaining strategy for single-issue negotiation}

The process of single-issue negotiation is shown in Fig.1. The key step of the process is to assess the quoted price of opponents and put forward a counter price while it can't be accepted.

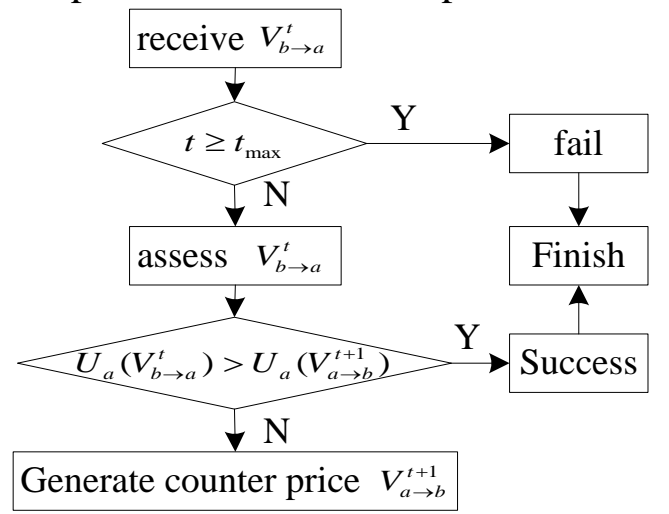

Fig. 1 process of single-issue negotiation

Calculate utility value of quoted price. Supposed that participants of bargaining negotiation is $A g=\{a, b\}$, where $a$ is buyer and $b$ is seller. Assumed that the quoted price of $a$ is $\left[P_{a}^{l}, P_{a}^{u}\right], P_{a}^{u}$ is the highest price can be accepted by $a$, while the quoted price of $b$ is $\left[\begin{array}{lll}P_{b}^{l}, & P_{b}^{u}\end{array}\right]$ and $P_{b}^{l}$ is the lowest price can be accepted by $b$. For $a$ and $b$, they don't know each other's reserve price. So, the interval $\left[P_{b}^{l}, P_{a}^{u}\right]$ as shown in Fig. 2 is the price able to make negotiation success.

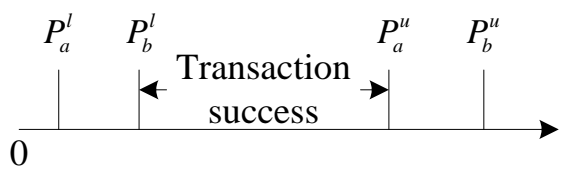

Fig. 2 interval makes transaction successful

As seller, $b$ usually wants to get the highest price, so its utility function is monotonically increasing. On the contrary, the utility function of $a$ is monotonically decreasing. So, the formula for calculating utility value of $t$-reincarnation bargaining can be defined as follow Eq. 2 and Eq. 3 respectively.

$$
\begin{aligned}
& U_{a}\left(V_{a \rightarrow b}^{t}\right)=\frac{P_{a}^{u}-V_{a \rightarrow b}^{t}}{P_{a}^{u}-P_{a}^{l}} \\
& U_{b}\left(V_{b \rightarrow a}^{t}\right)=\frac{V_{b \rightarrow a}^{t}-P_{b}^{l}}{P_{b}^{u}-P_{b}^{l}}
\end{aligned}
$$

Reincarnation number based bargaining strategy. Negotiation almost impossible to be successful immediately, it needs several rounds of bargaining. At the beginning of negotiation, $a$ and $b$ will present the price $V_{a \rightarrow b}^{1}$ and $V_{b \rightarrow a}^{1}$ makes their utility maximization. If negotiation fails, one of the participants will propose a new quoted price based on his concession strategy in subsequent negotiation. Supposed that the function of concession strategy is $f(t)$, the function of $a$ updates the price to $b$ in the next reincarnation can be defined as follow Eq. 4.

$$
x_{a \rightarrow b}^{t+1}=x_{a \rightarrow b}^{t}+f(t)\left(P_{a}^{u}-P_{a}^{l}\right)
$$


If $f(t)=1 / t_{\max }$, it means that the concession strategy of $a$ is uniform. $f(t)=1 / 2^{t-1}$ represents the concession strategy is monotonically decreasing and $f(t)=1 / 2^{t_{\max }-t}$ is on the contrary.

Rival behavior based bargaining strategy. The key of rival behavior based bargaining is to acquired the concession strategy from bargaining sequence of opponent's firstly, and then proposes a new price. Supposed that the bargaining sequence of $b$ to $a$ is $S_{b}=\left\{V_{b \rightarrow a}^{1}, V_{b \rightarrow a}^{2}, \ldots, V_{b \rightarrow a}^{t}\right\}$, then the concession strategy of $a$ can be defined in four different forms, as shown in Eq. 5.

$$
\left\{\begin{array}{l}
\Delta_{A A C D}^{b}=\frac{\left|V_{b \rightarrow a}^{1}-V_{b \rightarrow a}^{t}\right|}{t} \\
\Delta_{A M i n C D}^{b}=\min \left|V_{b \rightarrow a}^{i}-V_{b \rightarrow a}^{i-1}\right|, i=2,3, \ldots, t \\
\Delta_{A M a x C D}^{b}=\max \left|V_{b \rightarrow a}^{i}-V_{b \rightarrow a}^{i-1}\right|, i=2,3, \ldots, t \\
\Delta_{R A C D}^{b}=\frac{\left|V_{b \rightarrow a}^{t}-V_{b \rightarrow a}^{t-k}\right|}{k}
\end{array}\right.
$$

Where $V_{b \rightarrow a}^{1}$ is the initial quoted price of $b$ and $V_{b \rightarrow a}^{t}$ is the last quoted price of $b, t$ is the reincarnation number of negotiation.

According to $S_{b}$ and the concession strategy of $a$, the quoted price of $a$ in $t+1$-reincarnation can be calculated as follow Eq. 6 .

$$
V_{a \rightarrow b}^{t+1}=\min \left\{\max \left[V_{a \rightarrow b}^{t}-f\left(S_{b}\right), P_{a}^{l}\right], P_{a}^{u}\right\}
$$

Where $f\left(S_{b}\right)$ is concession strategy of $a$ calculated by $S_{b}$, and its value range is $f\left(S_{b}\right) \in\left\{\Delta_{A A C D}^{b}, \Delta_{A M i n C D}^{b}, \Delta_{A M a x C D}^{b}, \Delta_{R A C D}^{b}\right\}$.

\section{Bargaining strategy for multi-issue negotiation}

Calculate utility value of multi-issue. As to issues $\left.T=<T_{1}, T_{2}, \ldots, T_{n}\right\rangle$. Supposed that the weight of $a$ assigned to issue $T_{j}$ is $\omega_{j}^{a}$ where $\sum_{j=1}^{n} \omega_{j}^{a}=1 . U_{j}^{i}$ is utility values for issues $T_{j}$ in $i$-reincarnation. Then the formula for calculating utility value of multi-issue is defined as follow Eq.7.

$$
U^{a}=\sum_{j=1}^{n} \omega_{j}^{a} U_{j}^{i}
$$

Where

$$
\left\{\begin{array}{l}
U_{j}^{i}=\frac{V_{j}^{i}-P_{j}^{l}}{P_{j}^{u}-P_{j}^{l}}, \text { if } T_{j} \text { is gain issue } \\
U_{j}^{i}=\frac{P_{j}^{u}-V_{j}^{i}}{P_{j}^{u}-P_{j}^{l}}, \text { others }
\end{array}\right.
$$

And $\left[P_{j}^{l}, P_{j}^{u}\right]$ is the value interval of issue $T_{j}, V_{j}^{i}$ is practical value of $T_{j}$ in $i$-reincarnation.

Bargain strategy of equivalent utility value. As to multi-issues $T=<T_{1}, T_{2}, \ldots, T_{n}>$, if price $V_{a \rightarrow b}^{T}$ can't be accepted by $b$ in $i$-reincarnation negotiation, then $b$ will put forward a counter offer $V_{b \rightarrow a}^{T}$. If $U_{t}\left(V_{b \rightarrow a}^{T}\right) \geq U_{t+1}\left(V_{a \rightarrow b}^{T}\right)$, then $a$ accepts $V_{b \rightarrow a}^{T}$ and bargaining negotiation is success. Otherwise, $a$ will choose a counter-price $V_{a \rightarrow b}^{T}=\max \left\{\operatorname{sim}\left(V_{a \rightarrow b}^{T}, V_{b \rightarrow a}^{T}\right)\right\}$ while $U\left(V_{a \rightarrow b}^{T}\right)=U\left(V_{a \rightarrow b}^{T}\right)$.

As to the similarity between $V_{a \rightarrow b}^{T}$ and $V_{b \rightarrow a}^{T}$, it can be defined as follow Eq. 8.

$$
\operatorname{sim}\left(V_{a \rightarrow b}^{T}, V_{b \rightarrow a}^{T}\right)=\sum_{j=1}^{n}\left(\omega_{j}^{b} \times \operatorname{sim}\left(V_{a \rightarrow b}^{t_{j}}, V_{b \rightarrow a}^{t_{j}}\right)\right)
$$

Where $\operatorname{sim}\left(V_{a \rightarrow b}^{t_{j}}, V_{b \rightarrow a}^{t_{j}}\right)$ is similarity between the value from $a$ and $b$ on issue $t_{j}$ and it can be calculated by follow formula as shown in Eq. 9.

$$
\operatorname{sim}\left(V_{a \rightarrow b}^{t_{j}}, V_{b \rightarrow a}^{t_{j}}\right)=\frac{\min \left(V_{a \rightarrow b}^{t_{j}}, V_{b \rightarrow a}^{t_{j}}\right)}{\max \left(V_{a \rightarrow b}^{t_{j}}, V_{b \rightarrow a}^{t_{j}}\right)}
$$

The idea of proposing a price with equivalent utility value is divide issues $V^{T}=<V^{T_{1}}, V^{T_{2}}, \ldots, V^{T_{n}}>$ 
into $T^{1}=<T_{1}, T_{2}, \ldots, T_{m}>$ and $T^{2}=<T_{m+1}, T_{m+2}, \ldots, T_{n}>$ two sets firstly, where $m=[n / 2], T^{1} \cup T^{2}=T$ and $T_{1} \cap T_{2}=\varnothing$. In this way, $V_{a \rightarrow b}^{T}$ is divided into $V_{a \rightarrow b}^{T^{1}}=\left(V_{a \rightarrow b}^{T_{1}}, V_{a \rightarrow b}^{T_{2}}, \ldots, V_{a \rightarrow b}^{T_{m}}\right)$ and $V_{a \rightarrow b}^{T^{2}}=\left(V_{a \rightarrow b}^{T_{m+1}}, V_{a \rightarrow b}^{T_{T \rightarrow 2}}, \ldots, V_{a \rightarrow b}^{T_{n}}\right)$ correspondingly, and their utility is $U\left(V_{a \rightarrow b}^{T^{1}}\right)$ and $U\left(V_{a \rightarrow b}^{T^{2}}\right)$. Then the new price $V_{a \rightarrow b}^{T}$ can be acquired by the way of reducing utility value $U$ from $T^{1}$ and add the same utility value to $T^{2}$. The algorithm of reducing $U$ from $T^{1}$ is described as follow:

Input : $T^{1}=<T_{1}, T_{2}, \ldots, T_{m}>$ and its weight $W^{T^{1}}=\left\{\omega_{1}, \omega_{2}, \ldots, \omega_{m}\right\}$, decreased utility value $U$; $V_{a \rightarrow b}^{T^{1}}=\left(V_{a \rightarrow b}^{T_{i}}, V_{a \rightarrow b}^{T_{2}}, \ldots, V_{a \rightarrow b}^{T_{T}}\right)$ and $U\left(V_{a \rightarrow b}^{T_{i}}\right)=\left(V_{a \rightarrow b}^{T_{i}}-P_{T_{i}}^{l}\right) /\left(P_{T_{i}}^{u}-P_{T_{i}}^{l}\right)$

Output: $V_{a \rightarrow b}^{T^{1}}=\left(V_{a \rightarrow b}^{T_{1}}, V_{a \rightarrow b}^{T_{2}}, \ldots, V_{a \rightarrow b}^{T_{m}}\right)$

Init. : $k=0 ; U_{D}=0$

\section{Steps:}

1) for(int $i=1 ; i \leq m ; i++$ )

$$
U_{T_{i}}=U\left(V_{a \rightarrow b}^{T_{i}}\right)
$$

2) while $\left(U_{D}<U\right)$

$$
\begin{aligned}
& k=k+1 ; \\
& \text { for(int } i=1 ; \quad i \leq m ; i++) \\
& \qquad d_{i}^{k}=\operatorname{random}\left(0, U_{T_{i}}\right) ; \\
& U_{D}=U_{D}+\sum_{i=1}^{m} \omega_{i} d_{i}^{k} ; \\
& \text { for(int } i=1 ; i \leq m ; i++) \\
& \quad U_{T_{i}}=U_{T_{i}}-d_{i}^{k} ;
\end{aligned}
$$

3) for(int $i=1 ; i \leq m ; i++$ )

$$
U_{i}=\left(\sum_{j=1}^{k} \omega_{i} d_{i}^{j}\right)\left(U / U_{D}\right)
$$

4) for(int $i=1 ; i \leq m ; i++)$

$$
U\left(V_{a \rightarrow b}^{T_{i}}\right)=U\left(V_{a \rightarrow b}^{T_{i}}\right)-U_{i}
$$

Calculate $V_{a \rightarrow b}^{T_{i}}$ by $U\left(V_{a \rightarrow b}^{\prime T_{i}}\right)=\left(V_{a \rightarrow b}^{T_{i}}-P_{T_{i}}^{l}\right) /\left(P_{T_{i}}^{u}-P_{T_{i}}^{l}\right)$

5) Output $V_{a \rightarrow b}^{T^{1}}=\left(V_{a \rightarrow b}^{T_{1}}, V_{a \rightarrow b}^{T_{2}}, \ldots, V_{a \rightarrow b}^{T_{m}}\right)$, end.

The algorithm of add $U$ to $T^{2}$ is just add utility value for each issue randomly instead of reduce utility value in the above algorithm.

\section{Bargaining strategy based process of transaction in e-commerce}

Supposed that an enterprise $a$ has some tasks to be assigned to an outsource service supplier $b$ in the tasks applicants $B=\left\{b_{1}, b_{2}, \ldots, b_{r}\right\}$. The issues of bargaining negotiation between seller $a$ and supplier $b$ are cost, quality and delivery deadline. From the point view of $a$, the bargaining strategy based process of transaction can be described in Fig.3, and the process described as follow. 


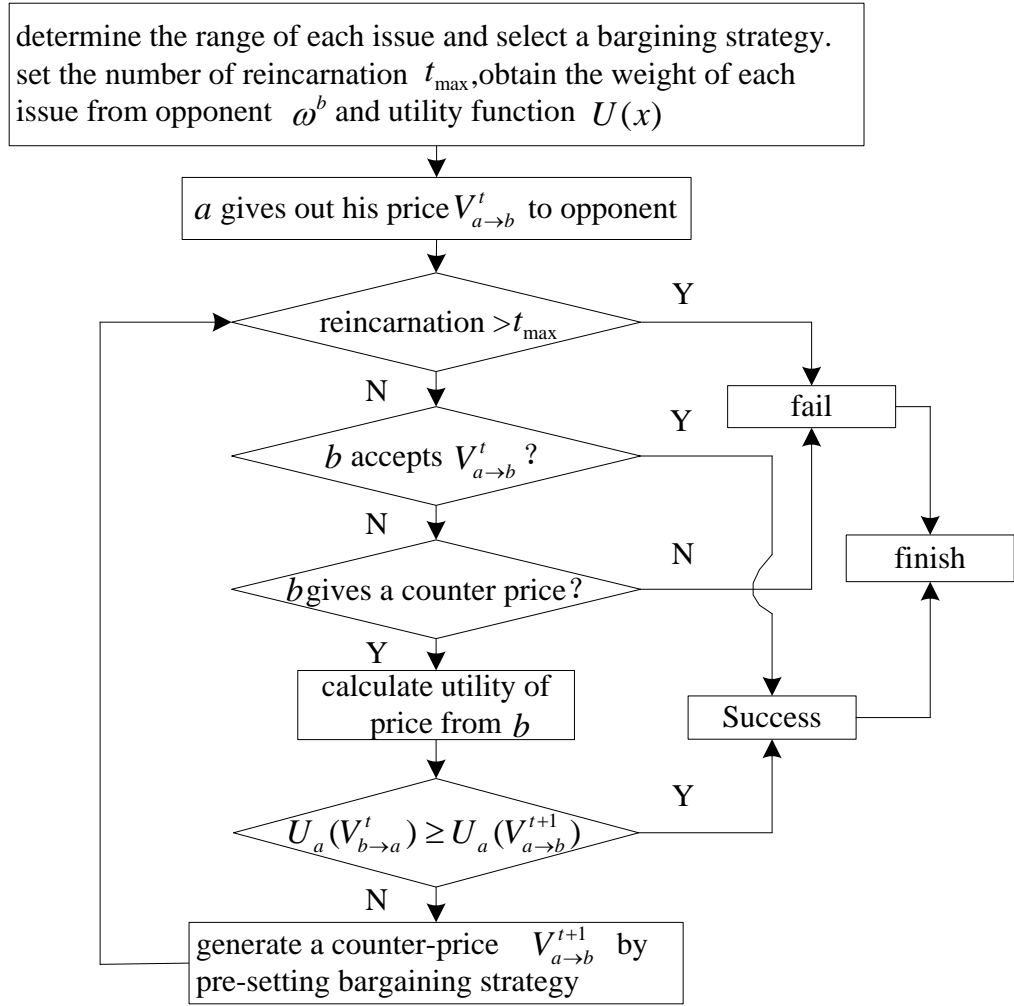

Fig.3 bargaining strategy based process of transaction

Step 1 Seller a determines the issues of negotiation and the range for each issue. Select a bargaining strategy and the utility function $U(x)$ for calculating utility value. Set the maximum value of reincarnation $t_{\max }$. If it is a multi-issue negotiation, seller $a$ also needs to know the weight of each issue from supplier $b$.

Step 2 Seller $a$ selects a supplier $b$ from $B=\left\{b_{1}, b_{2}, \ldots, b_{r}\right\}$ and proposes a price $V_{a \rightarrow b}^{t}$.

Step 3 If $b$ accepts $V_{a \rightarrow b}^{t}$, then turn step 7. If $b$ neither accepts it nor proposes a counter price, turn step 6. If supplier $b$ proposes a counter price, turn step 4 .

Step 4 Seller $a$ assesses the counter price from $b$ by $U(x)$ and turn step 7 while $U_{a}\left(V_{b \rightarrow a}^{t}\right) \geq U_{a}\left(V_{a \rightarrow b}^{t+1}\right)$. Otherwise, seller a proposes a counter price by the pre-setting bargaining strategy, then turn step 5.

Step 5 If the number of reincarnation $t=t_{\max }$, then turn step 6. Otherwise turn step 3.

Step 6 Negotiation fail, turn step 2.

Step 7 Negotiation success and tasks allocate to supplier $b$.

\section{Conclusions}

Negotiation is one of the best ways to resolve interest conflict in business negotiation in e-commerce, and bargaining strategy is the key of negotiation. For single-issue negotiation, the mainly used in bargaining is concession strategy, the reincarnation number based and bargaining behavior of opponents based bargaining can promote the success of negotiation effectively. While in multi-issue negotiation, the equivalent utility based bargaining can makes the negotiation parties to achieve a win-win situation.

\section{Reference}

[1] Ya'akov Gal. aamas'06 Proceedings of the fifth international joint conference on autonomous agents and multiagent system, p.370-376,( 2006). 
[2]Ma Benjiang, Qiu Wanhua. Journal of Industrial Engineering/Engineering Management, Vol.19, (2005), p. 123-125.

[3]Su Qin, Liu Qiang. Systems Engineering-Theory \& Practice,vol. 30, (2010), p.1002-1009.

[4]Wang Kanliang, Wang Song. Systems Engineering-theory \& Practice, vol.20, (2010), p. 1636-1642.

[5]Zhan Wenjie, Zou Yi. Systems Engineering—Theory \& Practice, vol.34, (2014), p.1181-1187.

[6]Li Yuyu, Huang Bo. Industrial Engineering Journal, vol.17,(2014), p.92-98.

[7]Wang Yi, Gong Yuan, Wang cheng. Proceedings of the CSU-EPSA, vol.25,(2013),p.136-142.

[8]Liu Yun, Feng Suiuli. Jourmal of Circuits and System,vol.17, (2012), p.21-26. 